\section{Additive Effect of Enterococcus faecium on Enterococcal Bloodstream Infections: A 14-Year Study in a Swiss
Tertiary Hospital}

\author{
Maja Weisser, MD; ${ }^{1}$ Selja Capaul, $\mathrm{PhD} ;{ }^{2}$ \\ Marc Dangel, MPH; ${ }^{1}$ Luigia Elzi, MD, MS; ${ }^{1}$ \\ Esther Kuenzli, MD, MSc; ${ }^{1}$ Reno Frei, MD; ${ }^{2}$ \\ Andreas Widmer, MD, MS, FIDSA, FSHEA ${ }^{1}$
}

\begin{abstract}
We investigated whether an increase in enterococcal bloodstream infections (BSIs) depends on the emergence of Enterococcus faecium in an area with low vancomycin-resistant enterococci prevalence. From 1999 to 2012 , a linear increase in E. faecium BSI rates $(0.009$ per 1,000 patient-days per year; $P<.001)$ was noted. Enterococcus faecalis BSI rates remained stable.
\end{abstract}

Infect Control Hosp Epidemiol 2013;34(10):1109-1112

In recent decades, enterococci have evolved from harmless intestinal commensals to important nosocomial pathogens worldwide.' In US hospitals during the 1980s, a shift from ampicillin-sensitive Enterococcus faecalis to ampicillin-resistant Enterococcus faecium (AREfm) was noted. Nowadays, vancomycin-resistant enterococci (VRE) account for more than $25 \%$ of enterococcal bloodstream infections (BSIs). ${ }^{2}$ In Europe, however, AREfm and vancomycin-resistant $E$. faecium. (VREfm) have been observed in clinical isolates only since $2000,{ }^{3}$ with large differences in the prevalence of VRE. In Switzerland, very low VRE rates are reported (less than $5 \%){ }^{4}$ The emergence of AREfm without an increasing incidence of VREfm of the VanA/B type in our institution allowed us to test the hypothesis that AREfm is primarily responsible for the overall increase in enterococcal infections.

\section{METHODS}

We retrospectively analyzed all routine blood cultures with growth of enterococci from patients hospitalized at the University Hospital Basel (UHBS), Switzerland, an 800-bed tertiary care teaching hospital, from January 1999 to December 2012. Data were extracted from the microbiological database and cross-checked by analysis of an independent backup system from the microbiology laboratory mainframe computer (ML Query DORNER EDV Systeme, Mephisto DB, HyBase).

From medical charts, all patients with enterococcal bacteremia were evaluated for the presence of a BSI. Two of the following criteria qualified for BSI: temperature more than $38^{\circ} \mathrm{C}$ or less than $36^{\circ} \mathrm{C}$, heart rate more than 90 per minute, tachypnea more than 20 per minute, and leukocytes either more than $12 \times 10^{9} / \mathrm{L}$ or less than $4 \times 10^{9} / \mathrm{L}$ (or more than $10 \%$ immature neutrophils). Nosocomial BSI was defined as BSI occurring more than 48 hours after hospital admission. Only the first enterococcal BSI per patient per year was included. BSI rates were calculated with respect to species differentiation (E. faecalis, E. faecium, other enterococci, Enterococcus spp.) expressed per 1,000 patient-days. The study was approved by the local ethical committee (EKBB 208/09).

Enterococcal species identification was performed by Vitek 2 (bioMérieux). Susceptibility testing was done for the first positive isolate of a patient's BSI. Minimal inhibitory concentrations (MICs) for ampicillin, ciprofloxacin, and vancomycin were performed by a semiautomated microdilution assay system (Micronaut; Merlin). Susceptibility to high-level gentamicin was tested using the Etest ( $\mathrm{AB}$ Biodisk; later bioMérieux). MICs were interpreted according Clinical and Laboratory Standards Institute guidelines until 2011 and according to European Committee on Antimicrobial Susceptibility Testing thereafter. From February to October 2008, 20 consecutive E. faecium blood isolates were typed with multilocus sequence typing (MLST) and multiple locus variable number tandem repeat analysis (MLVA), as described elsewhere. ${ }^{5,6}$

For comparison of the changes in infection rates, a linear regression model was used. A $P$ value of less than .05 was considered to indicate statistical significance.

\section{RES U L T S}

During the study period, of 22,518 positive blood cultures, $1,179(5.2 \%)$ from 453 patients grew enterococci. Of these, 424 originated from hospitalized patients, and 404 had a BSI. Species identification revealed 160 E. faecium (39.6\%), 215 E. faecalis (53.2\%), 18 other enterococci (4.5\%), and 22 nonspecified isolates (5.4\%). In 11 patients, two different enterococcal species were present simultaneously.

Over the study period, a linear increase in the rate of enterococcal BSI of 0.01 per 1,000 patient-days per year was observed $(P<.001$; Figure 1$)$. For $E$. faecium, the increase was 0.009 per 1,000 patient-days per year $(P<.001)$, manifest mostly after 2004 . For $E$. faecalis, the rate was stable over time (median, 0.064 per 1,000 patient-days; range, 0.0310.091; $P=.153$ ). Overall, 267 (66\%) BSIs were nosocomial. Whereas the rate of nosocomial E. faecium BSI increased over the study period, the rates of community-acquired $E$. faecium BSI and of nosocomial and community-acquired $E$. faecalis BSI remained stable. Most patients with a nosocomial $E$. faecium BSI were hospitalized in the intensive care unit or the hematology ward. On the hematology ward, the contribution of E. faecium BSI increased from $0 \%$ before 2004 to $22 \%$ of BSIs with any pathogen in 2012 (data not shown).

During the study period, only 1 BSI with a VRE VanB was 


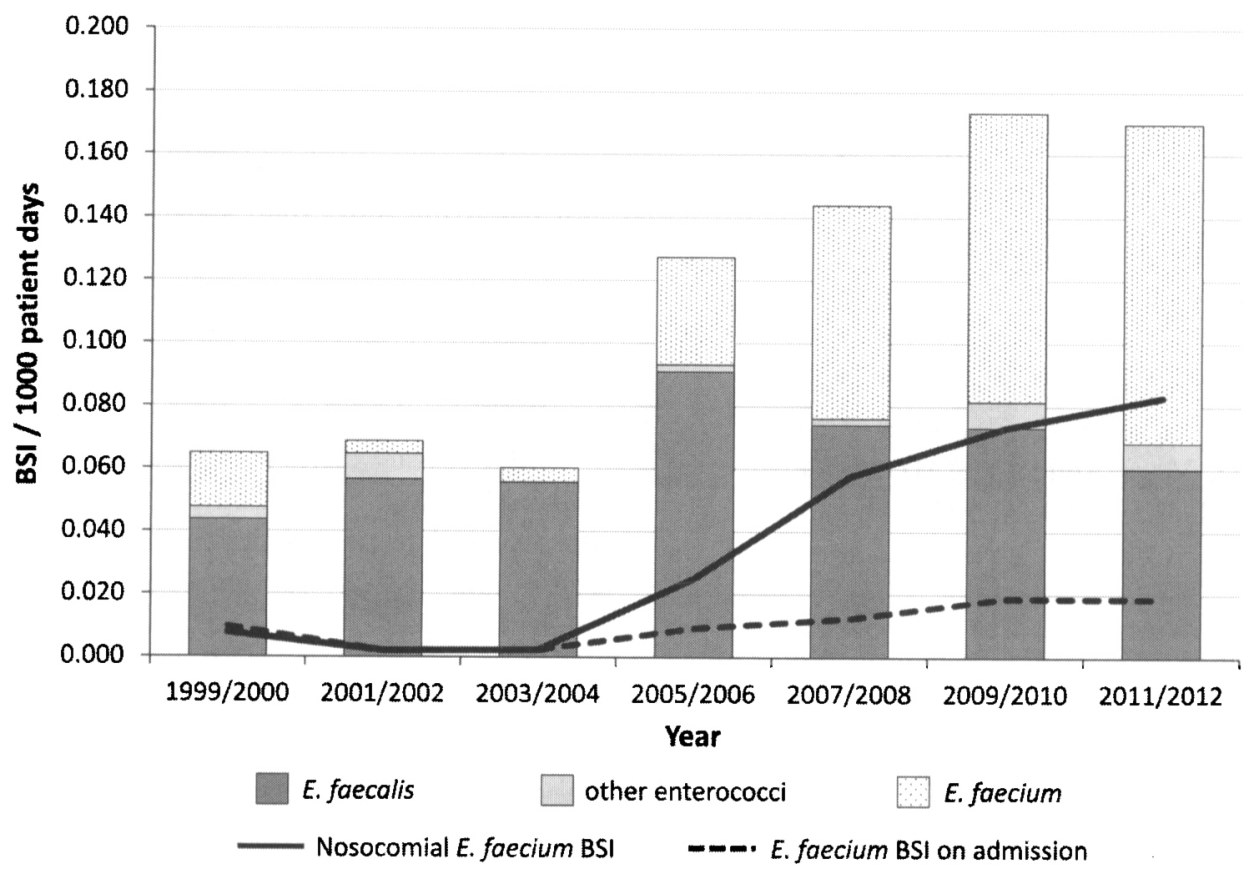

FIGURE 1. Bloodstream infections (BSIs) with enterococci (cases per 1,000 patient-days). Bars indicate BSIs with different enterococcal species per 1,000 patient-days during the period 1999-2012. Other enterococci consisted of E. casseliflavus $(n=8)$, E. durans $(n=3)$, E. avium $(n=3)$, E. gallinarum $(n=3)$, and E. hirae $(n=1)$. Lines indicate E. feacium BSIs per 1,000 patient-days during the period 1999-2012 according to nosocomial and community-acquired origin.

noted, in 2002. Among E. faecium isolates, ampicillin resistance rose from $0 \%$ in 1999 to $100 \%$ in 2012 in 1 and 14 tested isolates, respectively, with important fluctuations over time, mainly due to small numbers (Figure 2). The frequency of resistance to high-level gentamicin increased from $0 \%$ to $58 \%$. Resistance of E. faecium to quinolones increased slightly. $E$. faecalis isolates remained constantly susceptible to ampicillin and vancomycin and showed low rates of resistance to ciprofloxacin and high-level gentamicin.

MLST of 20 consecutive AREfm BSI isolates revealed 15 isolates with sequence type (ST) 78, 1 with ST192, and 1 with ST168, all corresponding to MT159 in MLVA. One isolate with ST168 corresponded to MT39. In 2 isolates (ST94 and ST22), no full MLVA profile was obtained, and no MT could be assigned. Resistance to ampicillin was present in 19 of these 20 isolates.

\section{DISCUSSION}

The increase in E. faecium BSIs over the 14-year period documented in our study reflects the change in global enterococcal epidemiology as described in other studies. ${ }^{3}$ In contrast to what has been thought previously, in our study $E$. faecium did not replace E. faecalis but was introduced newly into the nosocomial setting, similar to the emergence of methicillinresistant Staphylococcus aureus, which occurred on top of methicillin-susceptible $S$. aureus. ${ }^{7}$ This has also recently been shown for other nosocomial pathogens. ${ }^{8}$
The typing results of our study, although performed only during a short time period, document the presence of a known hospital-adapted epidemic clone. MLST ST78 belongs to the so-called clonal complex 17, after the presumed founder ST17. These clones-observed in many hospitals worldwide irrespective of the presence of VRE-are characterized by acquisition of multiple virulence and resistance genes. ${ }^{9}$ Whether they originate from cross-transmission in the hospital environment or whether preexisting AREfm outgrow the microbiome under strong selection pressure (eg, antibiotics) has not been proven yet, and it might be a multifaceted process, but data suggest transmission of a limited number of highly related AREfm clones within wards. ${ }^{3}$

Our study confirms reported resistance rates from the Swiss Center for Antibiotic Resistance (anresis). For the year 2011, published resistance rates for nosocomial $E$. faecium isolates were $86 \%$ for ampicillin ( $n=1,680), 37 \%$ for high-level gentamicin $(n=481)$, and $3 \%$ for vancomycin $(n=1,151){ }^{4}$ Despite the low prevalence of VRE, the possibility of integration of vanA and $\operatorname{van} B$ into a suitable "genetic background" is a major concern. The first outbreak of 31 VREfm was reported in 2011 in western Switzerland. ${ }^{10}$

Whether strict infection control measures, as recommended for MRSA or VRE, are effective and feasible for AREfm remains a matter of debate. Enterococci persist in patient environments for long time periods and are notoriously difficult to eradicate. ${ }^{3}$ Intensified infection control 

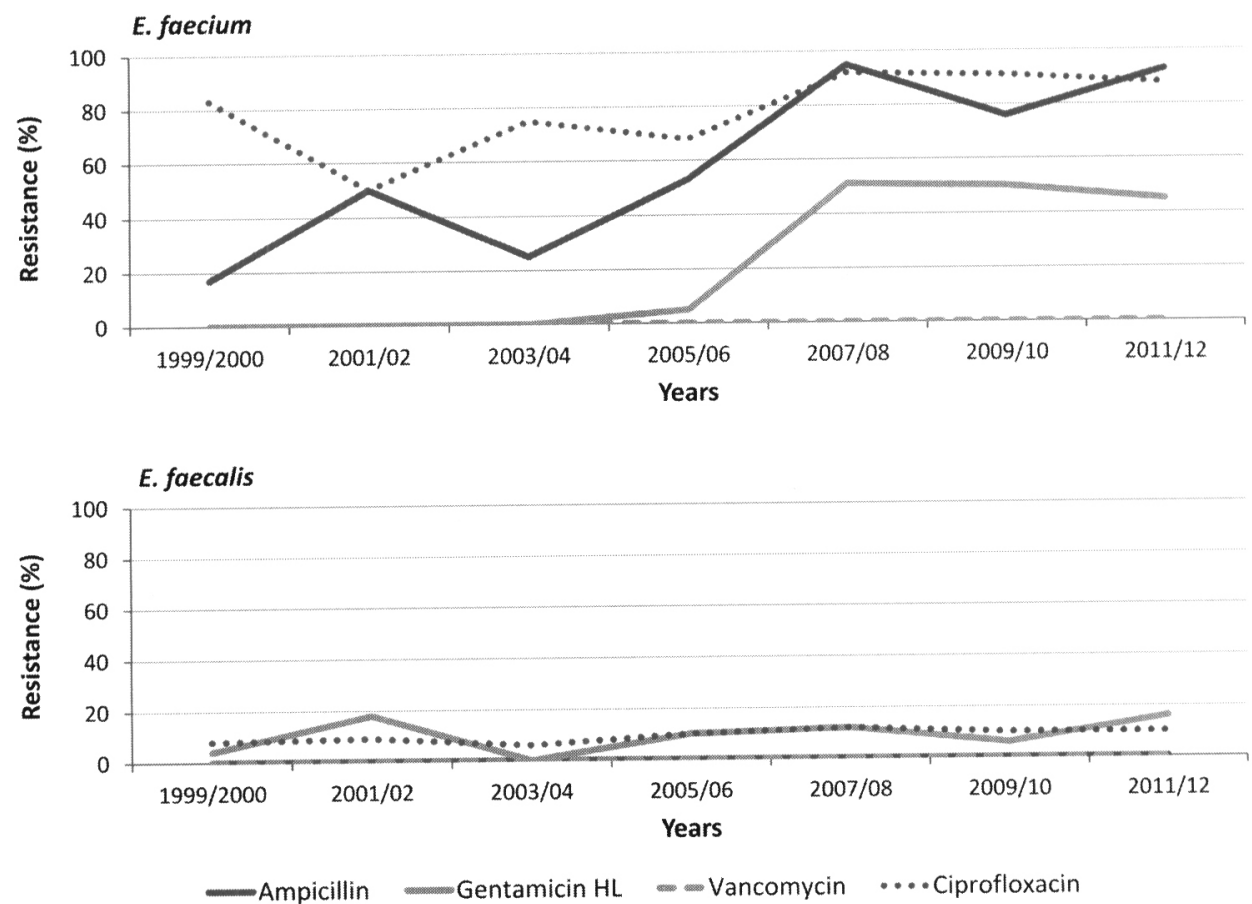

FIGURE 2. Resistance in Enterococcus faecium and Enterococcus faecalis from blood isolates. Lines show the percentage of resistant isolates (resistant isolates/tested isolates) among all E. faecium and E. faecalis isolates for the different antibiotics over time. HL, high level.

might be useful during a hospital outbreak. Its usefulness in a slowly changing epidemiology is unclear.

The strength of our study lies in the long observation period with data available on clinically relevant BSI. A limitation is the single-center study design, which makes extrapolation to other institutions difficult. Furthermore, typing data are limited to a short time period of the whole study, so it is possible that outbreaks with different types were missed.

In conclusion, we confirmed the emergence and gradual increase of an epidemic nosocomial $E$. faecium clone in BSI on top of a stable rate of infections with $E$. faecalis and community-acquired $E$. faecium. The nosocomial $E$. faecium clones belonging to $\mathrm{CC} 17$ might be the basis for the emergence of VRE.

\section{ACKNOWLEDGMENTS}

Financial support. This work was supported by the Stiftung Forschung Infektionskrankheiten (SFI 30), Freiwillige Akademische Gesellschaft (FAG), Basel, and Margarethe und Walter Lichtenstein-Stiftung, Universität Basel, Basel, Switzerland.

Potential conflicts of interest. All authors report no conflicts of interest relevant to this article. All authors submitted the ICMJE Form for Disclosure of Potential Conflicts of Interest, and the conflicts that the editors consider relevant to this article are disclosed here.

Affiliations: 1. Division of Infectious Diseases and Hospital Epidemiology, University Hospital Basel, Basel, Switzerland; 2. Division of Clinical Micro- biology, Department of Laboratory Medicine, University Hospital Basel, Basel, Switzerland.

Address correspondence to Maja Weisser, MD, Division of Infectious Diseases and Hospital Epidemiology, University Hospital Basel, Petersgraben 4, 4031 Basel, Switzerland (weisserm@uhbs.ch).

Received March 15, 2013; accepted May 15, 2013; electronically published August 29, 2013.

(C) 2013 by The Society for Healthcare Epidemiology of America. All rights reserved. 0899-823X/2013/3410-0013\$15.00. DOI: $10.1086 / 673145$

\section{REFERENCES}

1. Moellering RC Jr. Emergence of Enterococcus as a significant pathogen. Clin Infect Dis 1992;14(6):1173-1176.

2. Murdoch DR, Mirrett S, Harrell LJ, Monahan JS, Reller LB. Sequential emergence of antibiotic resistance in enterococcal bloodstream isolates over 25 years. Antimicrob Agents Chemother 2002;46(11):3676-3678.

3. de Regt MJ, van der Wagen LE, Top J, et al. High acquisition and environmental contamination rates of $\mathrm{CC} 17$ ampicillinresistant Enterococcus faecium in a Dutch hospital. I Antimicrob Chemother 2008;62(6):1401-1406.

4. Swiss Center for Antibiotic Resistance (anresis). anresis annual report 2013. Bern: anresis, 2013.

5. Homan WL, Tribe D, Poznanski S, et al. Multilocus sequence typing scheme for Enterococcus faecium. I Clin Microbiol 2002; 40(6):1963-1971.

6. Top J, Schouls LM, Bonten MJ, Willems RJ. Multiple-locus variable-number tandem repeat analysis: a novel typing scheme to study the genetic relatedness and epidemiology of Enterococcus faecium isolates. J Clin Microbiol 2004;42(10):4503-4511. 
7. Boyce JM, White RL, Spruill EY. Impact of methicillin-resistant Staphylococcus aureus on the incidence of nosocomial staphylococcal infections. J Infect Dis 1983;148(4):763.

8. Ammerlaan HS, Harbarth S, Buiting AG, et al. Secular trends in nosocomial bloodstream infections: antibiotic-resistant bacteria increase the total burden of infection. Clin Infect Dis 2013; 56(6):798-805.
9. Willems RJ, Top J, van Santen M, et al. Global spread of vancomycin-resistant Enterococcus faecium from distinct nosocomial genetic complex. Emerg Infect Dis 2005;11(6):821-828.

10. Senn L. Unprecedented nosocoimal spread of vancomycinresistant Enterococcus faecium in a tertiary-care hospital in Switzerland. Paper presented at: BMC Proceedings, 2011, Geneva. 